\section{A Fatal Case of Dengue-Associated Hemophagocytic Lymphohistiocytosis and Retroperitoneal Hematoma in a Patient With Autoimmune Hemolytic Anemia}

\author{
Chee Yik Chang ${ }^{1}$ \\ 1. General Medicine, Hospital Sultanah Aminah, Johor Bahru, MYS
}

Corresponding author: Chee Yik Chang, ccyik28@gmail.com

\begin{abstract}
Hemophagocytic lymphohistiocytosis (HLH) is a rare but potentially life-threatening complication of dengue infection which necessitates early diagnosis and treatment to improve patient outcomes. Severe dengue infection complicated by HLH may require interventions such as systemic corticosteroids, intravenous immunoglobulin, or chemotherapy. Here, we report a case of concurrent dengue-induced HLH and flare of underlying autoimmune hemolytic anemia (AIHA). The disease was refractory to treatment including corticosteroids and intravenous immunoglobulin. The course of illness was later complicated by dengue shock syndrome, severe liver dysfunction, and a large retroperitoneal hematoma. Unfortunately, the patient succumbed on day 10 of illness.
\end{abstract}

Categories: Internal Medicine, Infectious Disease, Hematology

Keywords: dengue fever, hemophagocytic lymphohistiocytosis, autoimmune hemolytic anemia, retroperitoneal hematoma, dengue shock syndrome

\section{Introduction}

Dengue virus is a single-stranded positive-sense ribonucleic acid virus and belongs to the family Flaviviridae, genus Flavivirus. The dengue virus has four distinct serotypes, namely dengue virus type 1 (DEN-1), DEN-2, DEN-3, and DEN-4 [1]. In recent years, there have been increasing cases of hemophagocytic lymphohistiocytosis (HLH) associated with dengue infection, leading to high mortality rates [2]. HLH is a hyperinflammatory condition that is characterized by macrophage activation with phagocytosis of blood cells in the bone marrow and subsequent cytokine storm, leading to organ dysfunction and death $[2,3]$.

HLH rarely occurs in the presence of autoimmune hemolytic anemia (AIHA). Wang et al. reported an unusual case of pregnancy-induced HLH with concomitant AIHA that resolved spontaneously after the termination of pregnancy [4]. Thus far, there has been no reported case on AIHA and HLH following dengue infection. Here, we report a case of severe dengue complicated by acute hemolysis with underlying warm AIHA, HLH, and retroperitoneal hematoma.

\section{() Copyright 2021}

Chang. This is an open access article distributed under the terms of the Creative Commons Attribution License CC-BY 4.0., which permits unrestricted use, distribution, and reproduction in any medium, provided the original author and source are credited.

\section{Case Presentation}

A 59-year-old lady with non-insulin-dependent diabetes mellitus presented to Hospital Sultanah Aminah, Johor, Malaysia with fever, myalgia, and headache of two days duration. She reported no bleeding tendency or rash. She had a known history of warm AIHA diagnosed two years prior. Her condition was deemed to be in remission during the last hematology clinic follow-up. She was on oral prednisolone $5 \mathrm{mg}$ daily and azathioprine $50 \mathrm{mg}$ daily.

Upon arrival to the hospital, she appeared alert and pale. Her blood pressure was 103/55 $\mathrm{mmHg}$ and her heart rate was 90 beats per minute. She was febrile, not tachypnoeic, and the oxygen saturation was $98 \%$ while breathing ambient air. Her initial full blood count showed a hemoglobin of $7.2 \mathrm{~g} / \mathrm{dL}$, while the white cell and platelet counts were within the normal limits. Due to the clinical suspicion of dengue infection, a rapid dengue non-structural (NS)-1 antigen test (SD Bioline Dengue Duo kit, Standard Diagnostics Inc., Seoul, Korea) was requested and shown to be positive, while dengue immunoglobulin M (IgM) and IgG were negative. Dengue virus type 3 (DEN-3) was later identified by reverse transcription-polymerase chain reaction (RT-PCR) assay. Peripheral blood film examination revealed active hemolysis with elevated reticulocyte count. The liver transaminases were elevated (alanine aminotransferase (ALT) 70 U/L, aspartate aminotransferase (AST) $137 \mathrm{U} / \mathrm{L}$ ) while the renal function test was normal. The serum ferritin and lactate dehydrogenase (LDH) on admission were $16851 \mathrm{mcg} / \mathrm{L}$ and $655 \mathrm{U} / \mathrm{L}$, respectively.

In the ward, the patient received maintenance intravenous fluid therapy $(1-1.5 \mathrm{cc} / \mathrm{kg} / \mathrm{hour})$ and she was started on intravenous hydrocortisone $100 \mathrm{mg}$ every eight hours in view of active AIHA. Since admission, 


\section{Cureus}

she was noted to be febrile until attaining defervescence on day five of illness. She remained normotensive and did not exhibit any signs of plasma leakage or hyperinflammation. Subsequently, the hematological and biochemical indices suggested reducing hemolysis.

However, her fever recurred on day 6-7 of illness with a high temperature recorded at $38.9^{\circ} \mathrm{C}$. Serial blood cultures were negative and the C-reactive protein level was not raised. At this point in time, the liver function tests were markedly deranged (ALT $281 \mathrm{U} / \mathrm{L}$, AST $1426 \mathrm{U} / \mathrm{L}, \mathrm{LDH} 3793 \mathrm{U} / \mathrm{L}$ ) and the serum ferritin level was $>100000 \mathrm{mcg} / \mathrm{L}$. Besides, hypofibrinogenemia and high triglyceride levels were also observed (Table 1). There was no organomegaly noted on physical examination. As a result, a clinical diagnosis of HLH was made as the calculated HScore was 197, with an 84\% probability of HLH [5]. Intravenous hydrocortisone was then changed to intravenous methylprednisolone $500 \mathrm{mg}$ daily, and intravenous $\mathrm{N}$ acetylcysteine was also started.

\begin{tabular}{|c|c|c|c|c|c|c|c|c|c|}
\hline & \multicolumn{9}{|c|}{ Day of illness } \\
\hline & D2 & D3 & D4 & D5 & D6 & D7 & D8 & D9 & D10 \\
\hline Haemoglobin (g/L) (12-15) & 7.2 & 7.6 & 7.4 & 7.0 & 8.1 & 8.5 & 9.9 & 7.0 & 6.3 \\
\hline Haematocrit (36-46) & 17 & 19 & 18 & 17 & 22 & 23 & 22 & 20 & 19 \\
\hline White cell $\left(\times 10^{9} / L\right)(4-10)$ & 4.6 & 3.9 & 5.8 & 4.7 & 3.6 & 3.8 & 3.6 & 6.3 & 10 \\
\hline Platelet $\left(x 10^{9} / \mathrm{L}\right)(150-410)$ & 178 & 166 & 127 & 127 & 105 & 94 & 51 & 49 & 74 \\
\hline ALT (U/L) (5-33) & 70 & 73 & 58 & 60 & 70 & 132 & 310 & 353 & 1006 \\
\hline AST (U/L) (5-32) & 137 & 148 & 187 & 233 & 267 & 525 & 1426 & 1609 & 4370 \\
\hline LDH (U/L) (135-214) & 655 & 837 & 1186 & 1555 & 1911 & 2054 & 3793 & 4954 & 5166 \\
\hline Total bilirubin $(\mu \mathrm{mol} / \mathrm{L})(<21)$ & 57 & 53 & 39 & 30 & 24 & 21 & 47 & 103 & 134 \\
\hline Direct bilirubin ( $\mu \mathrm{mol} / \mathrm{L})$ & 25 & & 18 & & 11 & & & & \\
\hline Indirect bilirubin ( $\mu \mathrm{mol} / \mathrm{L})$ & 32 & & 21 & & 13 & & & & \\
\hline Ferritin ( $\mu \mathrm{g} / \mathrm{L})(13-150)$ & 16851 & & & & & & $>100000$ & $>100000$ & $>100000$ \\
\hline Fibrinogen (g/L) (2.2-3.9) & & & & & & & 1.3 & 1.4 & 0.8 \\
\hline Triglyceride (mmol/L) (1.7-2.3) & & & & & & & 2.7 & 1.6 & 2.0 \\
\hline Creatinine $(\mu \mathrm{mol} / \mathrm{L})(44-80)$ & 45 & 49 & 41 & 36 & 39 & 33 & 30 & 49 & 96 \\
\hline
\end{tabular}

\section{TABLE 1: Serial blood investigations of the patient}

ALT: alanine aminotransferase; AST: aspartate aminotransferase; LDH: lactate dehydrogenase.

However, the patient did not improve despite three days of methylprednisolone with persistent fever, serial serum ferritin levels persistently $>100000 \mathrm{mcg} / \mathrm{L}$, and severe liver dysfunction. Therefore, intravenous immunoglobulin $4 \mathrm{mg} / \mathrm{kg}$ was given on day 8 of illness. Bone marrow examination was not performed due to her unstable condition. On day 9 of illness, she developed hypotensive shock that required multiple fluid boluses and inotropic support. She was subsequently intubated due to respiratory distress. She was noted to have increasing abdominal distension and a concurrent drop in hemoglobin of $3 \mathrm{~g} / \mathrm{dL}$ from the previous day (9.9 to $7.0 \mathrm{~g} / \mathrm{dL}$ ). Urgent abdominal ultrasonography revealed a large retroperitoneal hematoma measuring $10 \times 10 \times 14 \mathrm{~cm}$ (Figure 1). She was transfused with a total of eight units of packed cells and two cycles of disseminated intravascular coagulation (DIC) regimes. Her condition was deemed critically ill and unfit for surgical management of retroperitoneal hematoma. She finally succumbed on day 10 of illness. 


\section{Cureus}

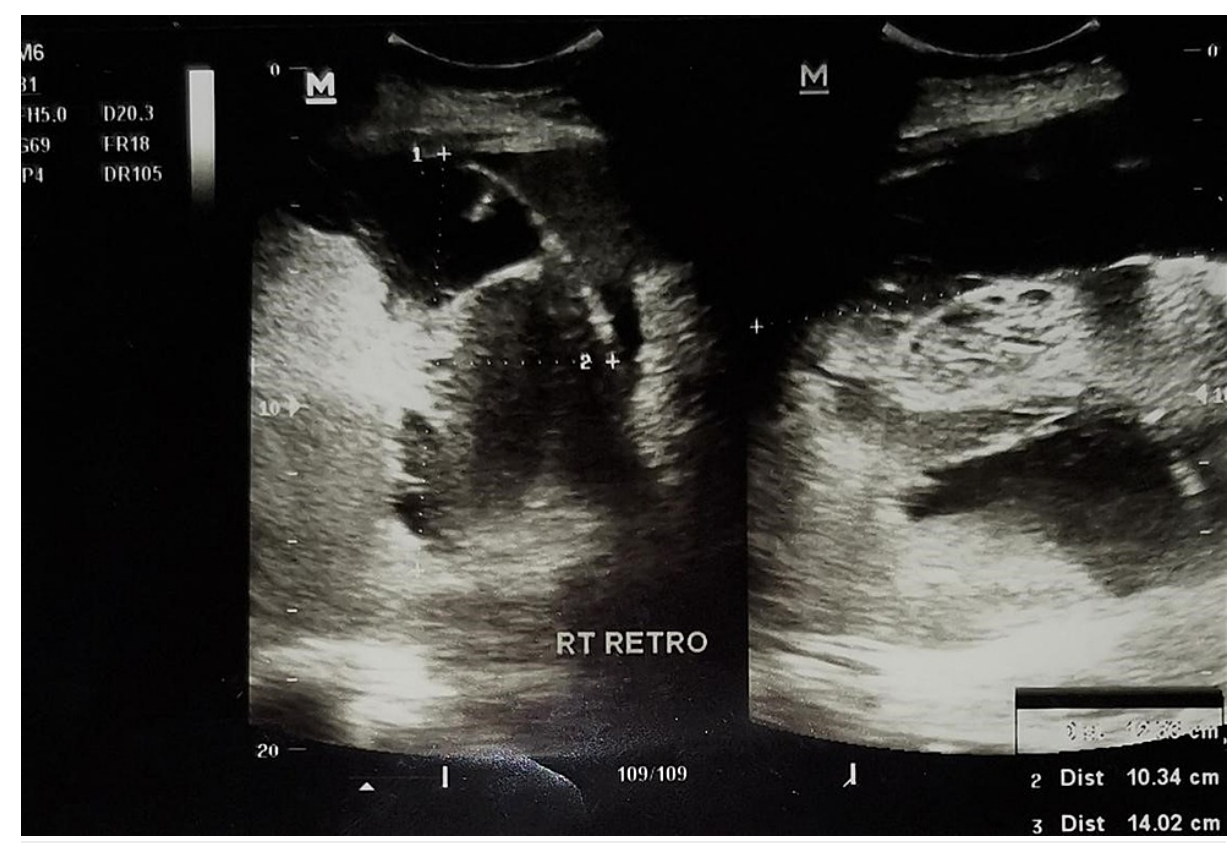

FIGURE 1: Abdominal ultrasonography showed a right retroperitoneal hematoma

\section{Discussion}

Dengue virus belongs to the genus flavivirus within the Flaviviridae family and is the cause of dengue infection. There are an estimated 50 million dengue infections and 500,000 individuals are hospitalized with dengue hemorrhagic fever each year, predominantly in Southeast Asia, the Pacific, and the Americas [1,6]. The mortality rate associated with dengue infection was reported to be around 20,000 deaths per year [6]. Severe dengue is the major cause of death resulting from plasma leakage, fluid accumulation causing respiratory distress, severe hemorrhage, or organ failure. Furthermore, severe dengue can be complicated by secondary HLH $[2,7]$.

Infection with the dengue virus has been increasingly recognized as an important cause of secondary HLH, and the mortality rate was as high as $43 \%[2,7]$. HLH is a hyperinflammatory condition that is characterized by macrophage activation with phagocytosis of blood cells in the bone marrow, and cytokine storm leading to organ dysfunction and death. To date, the exact mechanisms by which viruses are implicated in the pathogenesis of HLH remain unproven. Viruses may promote the development of HLH by evading immune recognition, inhibiting apoptotic pathways, and interfering with cytokine regulation [3]. HLH can be classified into primary (genetic) or secondary (acquired). The latter is caused by either a viral infection, autoimmune disease, or neoplastic condition [2]. Diagnosis of HLH requires fulfillment of at least five of the eight criteria as listed: fever, splenomegaly, cytopenia affecting at least two of three lineages in peripheral blood, ferritin $\geqslant 500 \mu \mathrm{g} / \mathrm{L}$, hypertriglyceridemia and/or hypofibrinogenemia, hemophagocytosis in bone marrow or spleen or lymph nodes, low or absent NK-cell activity, and an elevated level of soluble cluster of differentiation (CD) 25 [8].

Our patient had a history of warm AIHA for which she was on immunosuppressive therapy (prednisolone and azathioprine). AIHA is an uncommon disorder with an estimated incidence rate of 1-3 cases per 100000 per year and characterized by the production of antibodies directed against self-red blood cells $[9,10]$. We believed that the immunologic mechanisms involved in the pathogenesis of AIHA could predispose to HLH in this case. An unusual association between HLH, mixed connective tissue disease, and AIHA was reported previously by Kelkar et al. [11]. However, the development of HLH associated with dengue infection and AIHA has not been reported in the past. In this present case, dengue infection triggered an acute hemolytic episode in a patient with warm AIHA, and the course was then complicated by HLH. Despite early recognition of $\mathrm{HLH}$ and prompt treatment with corticosteroids and intravenous immunoglobulin, we were unable to reverse the severe, refractory HLH which eventually resulted in fatal outcome.

Dengue infection has various bleeding manifestations, ranging from asymptomatic petechial skin hemorrhage to life-threatening cerebral, pulmonary, and gastrointestinal hemorrhages [12]. Retroperitoneal hematoma is a very rare hemorrhagic complication of dengue infection and has only been described in several case reports $[12,13]$. 


\section{Conclusions}

HLH is an uncommon but severe complication in dengue infection whereby, in this case, AIHA might be an important predisposing factor. Early recognition and prompt treatment may improve patient outcomes in dengue-associated HLH. Severe dengue can be complicated by bleeding at the atypical site such as retroperitoneal hematoma. Hence, clinicians must be more vigilant of this severe, life-threatening complication in any patients presenting with abdominal distension or pain, rapid drop in hemoglobin, and dengue shock syndrome. Transfusion of blood products, correction of coagulopathy, and timely surgical intervention may improve outcomes.

\section{Additional Information \\ Disclosures}

Human subjects: Consent was obtained or waived by all participants in this study. Conflicts of interest: In compliance with the ICMJE uniform disclosure form, all authors declare the following: Payment/services info: All authors have declared that no financial support was received from any organization for the submitted work. Financial relationships: All authors have declared that they have no financial relationships at present or within the previous three years with any organizations that might have an interest in the submitted work. Other relationships: All authors have declared that there are no other relationships or activities that could appear to have influenced the submitted work.

\section{References}

1. Guzman MG, Halstead SB, Artsob H, et al.: Dengue: a continuing global threat . Nat Rev Microbiol. 2010, 8:S7-S16. 10.1038/nrmicro2460

2. Kan FK, Tan CC, Von Bahr Greenwood T, et al.: Dengue infection complicated by hemophagocytic lymphohistiocytosis: experiences from 180 patients with severe dengue. Clin Infect Dis. 2020, 70:2247-55. 10.1093/cid/ciz499

3. Brisse E, Wouters $\mathrm{CH}$, Andrei G, Matthys P: How viruses contribute to the pathogenesis of hemophagocytic lymphohistiocytosis. Front Immunol. 2017, 8:1102. 10.3389/fimmu.2017.01102

4. Wang LY, Hu J, Ramsingh G, Theodory B, Yaghmour B, Vergara-Lluri M, Yaghmour G: A case of recurrent pregnancy-induced adult onset familial hemophagocytic lymphohistiocytosis. World J Oncol. 2018, 9:123-7. 10.14740/wjon1145w

5. Fardet L, Galicier L, Lambotte O, et al.: Development and validation of the HScore, a score for the diagnosis of reactive hemophagocytic syndrome. Arthritis Rheumatol. 2014, 66:2613-20. 10.1002/art.38690

6. Stanaway JD, Shepard DS, Undurraga EA, et al.: The global burden of dengue: an analysis from the Global Burden of Disease Study 2013. Lancet Infect Dis. 2016, 16:712-23. 10.1016/S1473-3099(16)00026-8

7. Chang CY, Rajappan M, Zaid M, Ong ELC: Dengue fever complicated by hemophagocytic lymphohistiocytosis: report of 2 cases and bone marrow findings. Clin Case Rep. 2020, 8:3427-31. 10.1002/ccr3.3422

8. Henter JI, Horne A, Aricó M, et al.: HLH-2004: diagnostic and therapeutic guidelines for hemophagocytic lymphohistiocytosis. Pediatr Blood Cancer. 2007, 48:124-31. 10.1002/pbc.21039

9. Gehrs BC, Friedberg RC: Autoimmune hemolytic anemia. Am J Hematol. 2002, 69:258-71. 10.1002/ajh.10062

10. Barcellini W: New insights in the pathogenesis of autoimmune hemolytic anemia . Transfus Med Hemother. 2015, 42:287-93. 10.1159/000439002

11. Kelkar AH, Shah AA, Yong SL, Ahmed Z: An unusual association between hemophagocytic lymphohistiocytosis, mixed connective tissue disease, and autoimmune hemolytic anemia: a case report. Medicine. 2017, 96:e7488. 10.1097/MD.0000000000007488

12. Singh J, Singh H, Sukhija G, Jagota R, Bala S: Retroperitoneal haematoma in a patient with dengue haemorrhagic fever: a rare case report. J Clin Diagn Res. 2016, 10:OD01-OD02. 10.7860/JCDR/2016/20676.8771

13. Matthias AT, Apsara S, Epa A: A case report of dengue haemorrhagic fever complicated with psoas haematoma requiring blood transfusion. BMC Infect Dis. 2019, 19:385. 10.1186/s12879-019-4023-2 